\title{
Migraine in Canada... We Can and Should do Better
}

Can. J. Neurol. Sci. 2010; 37: 553-554

Historically, Headache Medicine has taken a backseat to other neurology subspecialties. It was just a few decades ago that migraine was considered by many to be simply a disease of 'neurotic women'. However, in the last 20 years, significant advances in headache classification, genetics, pathophysiology and treatment have brought the field of Headache Medicine into the 21 st century. Nevertheless, there remains an astonishing lack of knowledge about migraine in Canada and this is reflected by the under-recognition of migraine in the general population and the under-diagnosis and under-treatment of migraine by physicians.

In this issue of the Canadian Journal of Neurological Sciences, Drs. Cooke and Becker report on the findings of the Canadian Migraine in Women Study ${ }^{1}$. This is the first population-based study of migraine in Canada since $1994^{2}$ and regrettably, little has changed in the interim with respect to migraine management in Canada. In 1994, O'Brien and colleagues determined that the prevalence of migraine in Canada was $7.8 \%$ in males and $24.9 \%$ in females and this was estimated to constitute 2.6 million adult women and 0.8 million adult men in Canada $^{2}$. Notably, only $46 \%$ of those respondents who met International Headache Society criteria for migraine had ever received a diagnosis of migraine from a physician ${ }^{2}$.

The Canadian Women and Migraine Study is a large population-based telephone survey carried out on behalf of Headache Network Canada, a non-profit organization dedicated to providing headache-related education to migraine sufferers and the general public ${ }^{1}$. In this survey, $26 \%$ of all respondents reported experiencing headache symptoms that met International Headache Society criteria for the diagnosis of migraine headache in the last six months. This represents approximately four million Canadian women. These numbers are not surprising as they are very much in-line with previous Canadian studies. What is both surprising and troubling is that of those women in the Canadian Women and Migraine Study identified with migraine, only $51 \%$ had consulted a physician about their headaches.

There are several potential reasons why an individual with migraine might not consult a physician about her (or his) migraine headaches. First, there continues to be the perception in the general population that migraines are simply a nuisance/ annoyance and not a genuine medical problem warranting consultation with a physician. Second, in today's climate of primary care medicine, patients are often faced with a "one problem per appointment" policy. As such, headaches often are forced to take a backseat to other problems of perceived greater importance. Third, for many, there is a sense of learned therapeutic nihilism - if an individual's parent had either never sought or never received effective therapeutic management for their migraines then the daughter (or son) is often destined to perpetuate this therapeutic nihilism. Fourth, unfortunately, a not insignificant number of Canadians are medical "orphans" without a primary care physician. Fifth, there are numerous allied health professionals willing and interested to see and manage individuals with headaches (including chiropractors, naturopaths, osteo-paths, massage therapists etc.) and they actively solicit such individuals. Finally, and fortunately many individuals do indeed achieve satisfactory migraine management from available over-the-counter treatment options.

In terms of migraine management, in the Canadian Women and Migraine Study, $90 \%$ of migraine sufferers utilized medications to treat their headaches. Surprisingly, despite two decades of experience with migraine-specific triptans and despite the availability of numerous safe, well-tolerated and efficacious triptans in Canada (there are seven triptans approved in Canada), only $8 \%$ of survey respondents were using triptans as their principal migraine medication. Notably, respondents were almost $300 \%$ more likely to be using codeine-containing analgesics and were almost $500 \%$ more likely to be using overthe-counter ibuprofen as their principal migraine medication (as opposed to a triptan). Given the significant personal, societal and health-care burden created by too frequent use of narcotic analgesics and given the potential for serious medicationinduced gastrointestinal and renal side-effects from unfettered use of over-the-counter NSAIDs the fact that only $8 \%$ of migraineurs are using triptans is quite disconcerting.

When queried about the psychosocial burden of their migraine headaches, $73 \%$ of migraineurs in the Migraine in Women Study indicated that they sometimes feel a lack of control over their lives, $92 \%$ missed days at work or family activities and $75 \%$ experienced a lack of understanding or cynicism from those around them. Furthermore, the migraineurs in the study reported a high level of disability with at least partial incapacitation on an average of almost 21 days a year. Notably, frequent migraineurs in the study (which translates to approximately 1.2 million Canadian women) reported being partially or completely incapacitated by their migraines, on average 36.6 days per year! This migraine-related disability has previously been identified in similar studies around the world and migraine-related disability has been recognized and acknowledged by the World Health Organization. They consider migraine to be one of the top 20 disorders world-wide in terms of days of life lost due to disability ${ }^{3,4}$.

Canadian migraineurs deserve better. So, what can be done to facilitate positive transformation? Without question, progress begins with enhanced education.

First, publication education campaigns are needed in order to teach the public about migraine headache and to dispel popular misconceptions about migraine, sinus, tension-type, TMJ-related and other headache disorders. Neurologists with interest in headache and patient advocates need to spear-head these initiatives through multi-channel distributions (e.g.. television, print media, web-based media).

Second, physician-education is paramount. Most Canadian medical schools devote approximately two hours of their four- 
year curriculum to headache and these two hours are usually divided among important secondary headache disorders (subarachnoid hemorrhage, brain tumor, meningitis, temporal arteritis etc.) as well as primary headache disorders. This is clearly insufficient and unsatisfactory given that headache is the single most common symptom that humans' experience ${ }^{5}$ and a leading cause of physician and emergency department visits ${ }^{6,7}$ and a significant cause of workplace absenteeism and presenteeism (days present at work but with reduced productivity $)^{8}$. Importantly, most family medicine residency programs as well as neurology residency programs devote relatively little attention to headache-related education.

Despite the fact that headache is the most common reason for neurology consultation in Canada ${ }^{9}$, most neurology residents in Canada will complete their residency programs having never spent a day in a Headache Clinic and having only a cursory working knowledge of practical migraine management not to mention the management of the dozens of other less-common primary and secondary headache disorders. This knowledge gap needs to be improved and the Canadian Headache Society is working hard to develop plans to provide sorely needed knowledge transfer.

With their publication of the Canadian Women and Migraine Survey, Drs. Cooke and Becker remind us that the millions of Canadians with migraine deserve better. We can do better. We must do better. The time has come to move Headache Medicine from the position of poor second cousin of neurology subspecialties to the very prominent seat at the neurology head table that it rightly deserves.

\section{REFERENCES}

1. Cooke LJ, Becker WJ. Migraine prevalence, treatment and impact: the Canadian women and migraine study. Can J Neurol Sci. 2010; 37:580-7.

2. O'Brien B, Goeree R, Streiner D. Prevalence of migraine headache in Canada: a population-based survey. Int J Epidemiology. 1994; 23:1020-6.

3. Pryse-Phillips W, Findlay H, Tugwell, et al. A Canadian population survey on the clinical, epidemiologic and societal impact of migraine and tension-type headache. Can J Neurol Sci. 1992;19: 333-9.

4. Menken M, Munsat TL, Toole JF. The global burden of disease study: implications for neurology. Arch Neurol. 2000;57:418-20.

5. Hammond EC. Some preliminary findings on physical complaints from a prospective study of 1064004 men and women. Am J Public Health. 1964;54:11-23.

6. Cherry DK, Curt CW, Woodwell DA. National Ambulatory Medical Care Survey: 2001 summary. Advance Data. 2003;337:1-44.

7. McCaig LF, Burt CW. National Hospital Ambulatory Medical Care Survey 2001: emergency department summary. Advance Data. 2003;335:1-29.

8. Stewart WF, Ricci JA, Chee E, et al. Lost productive time and cost due to common pain conditions in the US workforce. JAMA. 290(18):2443-54.

9. Rajput AH, Uitti RJ, Rajput AH. Neurological disorders and services in Saskatchewan - a report based on provincial health care records. Neuroepidemiology. 1988;7:145-51.
Jonathan Gladstone

Toronto, Ontario, Canada

Paul E. Cooper

London, Ontario, Canada 